\title{
A gonadotropin-releasing hormone-like molecule modulates the activity of diverse central neurons in a gastropod mollusk, Aplysia californica
}

\author{
Biao Sun and Pei-San Tsai * \\ Department of Integrative Physiology, University of Colorado, Boulder, CO, USA
}

\section{Edited by:}

Gustavo M. Somoza, Instituto de Investigaciones BiotecnologicasInstituto Tecnologico de Chascomus, Argentina

Reviewed by:

Anna Di Cosmo, University of Naples Federico II, Italy

Nancy Sherwood, University of Victoria, Canada

Nancy Wayne, University of California Los Angeles, USA

\section{${ }^{*}$ Correspondence}

Pei-San Tsai, Department of

Integrative Physiology, University of

Colorado, 114 Clare Small, Boulder,

CO 80309-0354, USA.

e-mail:pei-san.tsai@colorado.edu
In vertebrates, gonadotropin-releasing hormone $(\mathrm{GnRH})$ is a crucial decapeptide that activates the hypothalamic-pituitary-gonadal axis to ensure successful reproduction. Recently, a GnRH-like molecule has been isolated from a gastropod mollusk, Aplysia californica. This $\mathrm{GnRH}$ (ap-GnRH) is deduced to be an undecapeptide, and its function remains to be explored. Our previous study demonstrated that ap-GnRH did not stimulate a range of reproductive parameters. Instead, it affected acute behavioral and locomotive changes unrelated to reproduction. In this study, we used electrophysiology and retrograde tracing to further explore the central role of ap-GnRH. Sharp-electrode intracellular recordings revealed that ap-GnRH had diverse effects on central neurons that ranged from excitatory, inhibitory, to the alteration of membrane potential. Unexpectedly, extracellular recordings revealed that ap-GnRH suppressed the onset of electrical afterdischarge in bag cell neurons, suggesting an inhibitory effect on female reproduction. Lastly, using immunocytochemistry coupled with nickel backfill, we demonstrated that some ap-GnRH neurons projected to efferent nerves known to innervate the foot and parapodia, suggesting ap-GnRH may directly modulate the motor output of these peripheral tissues. Overall, our results suggested that in A. californica, ap-GnRH more likely functioned as a central modulator of complex behavior and motor regulation rather than as a conventional reproductive stimulator.

Keywords: GnRH, Aplysia californica, sea hare, reproduction, electrophysiology, gastropod mollusk, central nervous system

\section{INTRODUCTION}

In vertebrates, gonadotropin-releasing hormone $(\mathrm{GnRH})$ is critical for the activation of the hypothalamo-pituitary-gonadal (HPG) axis and thus reproductive success (reviewed in Somoza et al., 2002). There is now evidence demonstrating that GnRH-like peptides are also present in non-chordate invertebrates (reviewed by Kah et al., 2007; Tsai and Zhang, 2008). A total of 26 variants of GnRH (Roch et al., 2011), including 2 from the mollusks (Iwakoshi et al., 2002; Zhang et al., 2008), have been identified. An additional four forms of GnRH-like molecules have been found in the owl limpet (Lottia gigantean), a polychaete worm (Capitella sp.), a sea urchin (Strongylocentrotus purpuratus), and a leech (Helobdella robusta) by searching within the expressed sequence tag database (Tsai and Zhang, 2008; Roch et al., 2011). GnRH from two species of cephalopod mollusk, common cuttlefish (Sepia officinalis; Di Cristo et al., 2009) and sword tip squid (Loligo edulis; Onitsuka et al., 2009) share the same peptide sequence as the common octopus (Octopus vulgaris; Iwakoshi et al., 2002). The isolated or deduced sequences of these GnRH peptides have either 11 or 12 amino acids with the insertion of 2 amino acids downstream of the N-terminal pyroglutamyl residue (Iwakoshi et al., 2002; Zhang et al., 2008; Di Cristo et al., 2009; Onitsuka et al., 2009; Tsai et al., 2010; Roch et al., 2011). The primary structure of these six novel forms of GnRHs differs markedly from that of chordate GnRHs, challenging the conventional belief that the decapeptide structure of chordate GnRHs is universally conserved. The diversification of GnRH structure further raises the possibility that non-chordate GnRH may have assumed diverse functions unrelated to reproduction during evolution (reviewed in Tsai and Zhang, 2008).

The physiological roles of two molluscan forms of GnRH have been examined previously in the O. vulgaris (reviewed in Minakata et al., 2009) and the sea hare (Aplysia californica; Tsai et al., 2010). In the octopus, early studies using heterologous vertebrate $\mathrm{GnRH}$ antisera suggested that $\mathrm{GnRH}$ played a role in the regulation of central nervous system (CNS) and reproductive tissues (Di Cosmo and Di Cristo, 1998; Di Cristo et al., 2002; Di Cristo and Di Cosmo, 2007). These findings are consistent with the results obtained from the native GnRH (oct-GnRH) which appeared to modulate reproductive function via the stimulation of oviductal contraction and gonadal steroid secretion (Kanda et al., 2006; Minakata et al., 2009). However, the wide distribution of oct-GnRH in the CNS of $O$. vulgaris also suggested a wide range of non-reproductive functions associated with motor and memory regulation (reviewed in Minakata et al., 2009). In A. californica, the native form of GnRH (ap-GnRH) acutely altered behaviors such as parapodial opening, substrate attachment, and feeding, but did not stimulate any reproductive parameters tested, including gametogenesis, gamete release, and the accumulation of a reproductive hormone, egg-laying hormone (ELH; Tsai et al., 2010). In this regard, the 
literature suggested that molluscan GnRH could modulate a wide range of motor behaviors unrelated to reproduction.

In the present study, we utilized extracellular and sharpelectrode intracellular recordings combined with retrograde labeling to explore the central effects of ap-GnRH that may underlie some of the behavioral changes observed previously (Tsai et al., 2010). In addition, we determined if ap-GnRH altered the ability of the neuroendocrine bag cell neurons (BCN) to undergo electrical afterdischarge (AD). As AD is a key event driving ELH secretion from BCN, and thus egg-laying (Kupfermann and Kandel, 1970; Wayne and Wong, 1994), it is a convenient target for reproductive modulation. Overall, our results strongly support a role of ap-GnRH as a modulator of activities in diverse central neurons. Further, ap-GnRH neurons project axons directly to nerves responsible for the motor innervation of the foot and the parapodia, suggesting a direct role in motor control. Lastly, to our surprise, ap-GnRH suppresses the ability of $\mathrm{BCN}$ to undergo $\mathrm{AD}$, reflecting an inhibitory role in female reproduction.

\section{MATERIALS AND METHODS ANIMALS}

Wild-caught A. californica were purchased from Alacrity Marine Biological Services (Redondo Beach, CA, USA) from January to August. All animals were kept in a 400-gal tank of Instant Ocean recirculated through biological and chemical filters. The water temperature was $15-18^{\circ} \mathrm{C}$. Animals were fed Romaine lettuce daily. All sexually mature animals received an injection of atrial gland extract, which induces egg-laying (Nagle et al., 1985), to ensure they are competent to lay eggs. All experimental animals were anesthetized humanely by an injection of $1 / 3$ body volume of ice-cold isotonic $\mathrm{MgCl}_{2}$ before sacrifice.

\section{GnRH AND OTHER REAGENTS}

Synthetic ap-GnRH (pQNYHFSNGWYA-NH ${ }_{2}$ ) was manufactured by Genscript (Piscataway, NJ, USA) with $>96 \%$ purity. All other chemicals were purchased from Sigma-Aldrich (St. Louis, MO, USA).

\section{ap-GnRH EXTRACTION}

Pedal nerves P1, P8, and P9, and pleurovisceral connective nerve (PVC; Figure 1) were collected from different animals and snapfrozen on dry ice. These three pedal nerves innervate primarily the pedal and parapodial areas (Jahan-Parwar and Fredman, 1978; Kandel, 1979), regions previously shown to be modulated by ap-GnRH (Tsai et al., 2010). The PVC connects the pleural to abdominal ganglia and transmits stimulatory signals to the $\mathrm{BCN}$ to elicit AD, but is not known to be motor (Kandel, 1979; Figure 1). Three pairs of the same nerves were pooled and extracted for ap-GnRH as described in Tsai et al. (2010). The extracts were stored at $-20^{\circ} \mathrm{C}$ until the measurement of ap- $\mathrm{GnRH}$ by a specific radioimmunoassay (RIA).

\section{ap-GnRH IODINATION AND RIA}

The iodination of ap-GnRH was performed as previously described (Tsai et al., 2010). The homologous ap-GnRH RIA has been validated previously and found to be highly specific for apGnRH (Tsai et al., 2010). The limit of detection and the intra- and

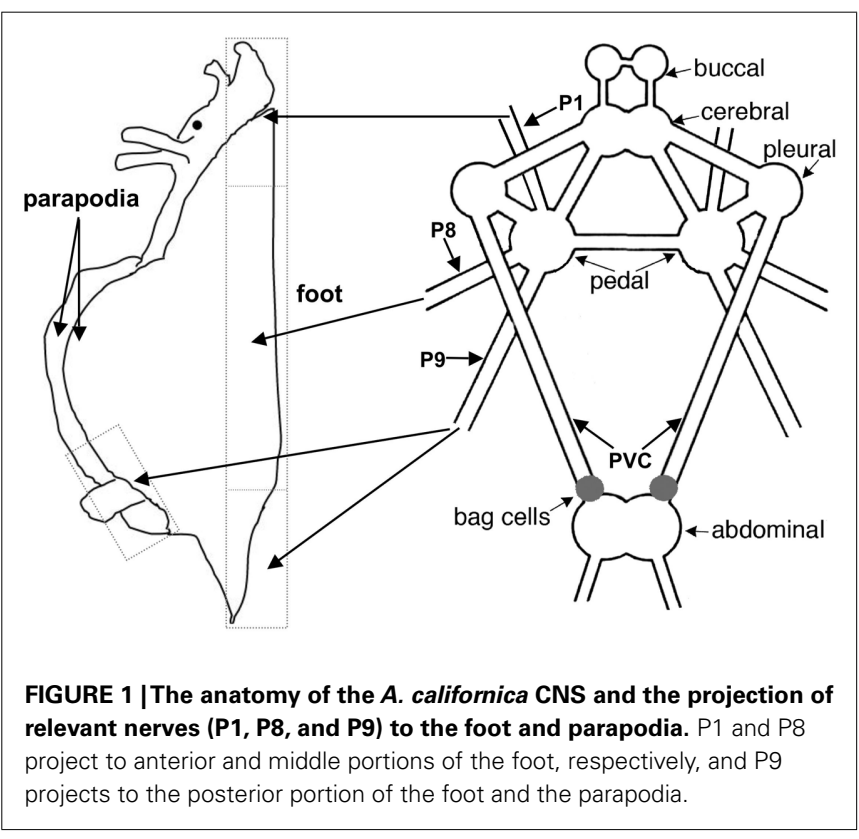

inter-assay coefficients of variation were $1.5 \mathrm{pg}, 7.1 \%$, and $8.5 \%$, respectively. All samples were assayed in five serially diluted doses.

\section{RETROGRADE LABELING BY NICKEL BACKFILL}

For nickel backfill, we employed a procedure modified from Fredman (1987). Briefly, pedal ganglia with right side nerves P1, P8, and P9 were pinned onto a Sylgard-lined dish containing sterile artificial seawater (ASW; $395 \mathrm{mM} \mathrm{NaCl}, 10 \mathrm{mM} \mathrm{KCl}, 10 \mathrm{mM} \mathrm{CaCl}_{2}$, $50 \mathrm{mM} \mathrm{MgCl} 2,28 \mathrm{mM} \mathrm{Na}_{2} \mathrm{SO}_{4}$, and $30 \mathrm{mM}$ HEPES) supplemented with $10 \%$ hemolymph. Individual nerves were trimmed to a length of about $1-1.5 \mathrm{~cm}$ and the cut ends shocked osmotically in distilled water for $20 \mathrm{~s}$. The cut ends were then placed in a chamber containing a nickel-lysine solution $\left(0.38 \mathrm{M} \mathrm{NiCl}_{2}\right.$ and $1.2 \mathrm{M} \mathrm{L}^{-}$ lysine). The chamber was sealed with petroleum jelly and the whole ganglia incubated at $15^{\circ} \mathrm{C}$ for $72 \mathrm{~h}$. The detection of $\mathrm{NiCl}_{2}$ uptake was carried out by incubating the ganglia in ASW containing $0.2 \%$ of a saturated dithiooxamide/dimethyl sulfoxide (DMSO) solution at room temperature for $1.5 \mathrm{~h}$. Afterward, the ganglia were pinned and immersion-fixed in Bouin's fixative overnight and stored in $70 \%$ ethanol. Fixed ganglia were dehydrated through ascending ethanol series, cleared in Histoclear II (National Diagnostics, Atlanta, GA, USA), embedded in paraffin, sectioned at $12-\mu \mathrm{m}$ thickness and mounted on gelatin-subbed slides. Sections were either immediately cleared and coverslipped or processed for ap-GnRH immunocytochemistry (ICC) described below.

\section{ap-GnRH ICC}

Immunocytochemistry was performed using a specific ap-GnRH antiserum (AS203-2) on sections of pedal ganglia after nickel backfill. The ICC procedure followed the protocol established previously (Tsai et al., 2003, 2010).

\section{EXTRACELLULAR RECORDING OF BCN}

The $\mathrm{AD}$ of $\mathrm{BCN}$ clusters from sexually mature animals competent to lay eggs was monitored in vitro to determine whether 
ap-GnRH modulated BCN activity (Zhang et al., 2000). Briefly, left and right BCN clusters with PVC attached were excised separately. $\mathrm{AD}$ was stimulated and recorded with suction electrodes placed on the PVC and distal BCN, respectively. In each study, one of the paired BCN clusters received vehicle (Veh) treatment $(0.0002 \%$ DMSO in ASW), and one received ap-GnRH treatment $(73 \mathrm{nM})$ for $30 \mathrm{~min}$. The application of ap-GnRH at $73 \mathrm{nM}$ was based on an earlier report in which $100 \mathrm{nM}$ GnRH2 elicited an inhibitory effect on the duration of bag cell AD (Zhang et al., 2000). Treatments were randomized between experiments so Veh and ap-GnRH treatments were applied to right and left BCN clusters the same number of times. After treatment, AD was initiated by a Grass S44 stimulator using the following stimulus parameters: $10 \mathrm{~V}$ at $6 \mathrm{~Hz}$ and $2.5 \mathrm{~ms}$ duration for $30 \mathrm{~s}$. BCN clusters were stimulated at an interval of $30 \mathrm{~s}$ until an $\mathrm{AD}$ was triggered. If no $\mathrm{AD}$ was triggered after five stimuli, BCN clusters were left to recover for $30 \mathrm{~min}$ before the second round of stimulus using the same protocol. BCN clusters failing to respond after the second round were judged as non-responsive. Compound action potentials $(\mathrm{AP})$ in an $\mathrm{AD}$ were monitored by a Grass amplifier interfaced with LabScribe2 software (iWorx Systems, Dover, NH, USA).

\section{INTRACELLULAR RECORDINGS}

The ganglia were glued to a glass coverslip and anchored to the floor of a Sylgard-coated recording chamber. The connective tissue surrounding the ganglia was manually desheathed to expose the neurons. Intracellular recordings were obtained with glass microelectrodes filled with $1.5 \mathrm{M} \mathrm{KCl}$ (resistance $=10-20 \mathrm{M} \Omega$ ). Signals were amplified and acquired with an AxoClamp 2B intracellular amplifier interfaced with LabScribe2 software. Only neurons with a resting membrane potential of $-40 \mathrm{mV}$ or lower, indicative of successful penetration and healthy cells, were included in the data analysis. All neurons recorded were noted for their relative sizes and for positions in the ganglia as superior, inferior, medial, lateral, dorsal, or ventral. These positions were used in our analysis to avoid the repeated inclusion of the same neurons from multiple recordings. Injections of depolarizing current were delivered with a S48 Grass stimulator to test evoked AP. A bridgebalanced circuit was used to adjust for the stimulus-induced voltage artifact.

After a successful penetration, the neuron was monitored for a few min until a stable voltage reading was obtained. Membrane potential was monitored during the following treatment sequence: Bath application of $0.002 \%$ DMSO (ap-GnRH vehicle) for $10 \mathrm{~min}$, followed by perfusion with ASW at $7 \mu \mathrm{l} / \mathrm{s}$ for $15 \mathrm{~min}$ to wash out the Veh, followed by the bath application of $730 \mathrm{nM}$ ap-GnRH for $10 \mathrm{~min}$. The choice of $730 \mathrm{nM}$ ap-GnRH was based on an earlier study (Goldberg et al., 1993) in which low micromolar range of mammalian $\mathrm{GnRH}(\mathrm{mGnRH})$ was shown to elicit diverse electrophysiological effects on central neurons of the pond snail, Helisoma trivolvis. At the end of 10-min Veh or ap-GnRH treatment period, a 2-s depolarizing current of $0.01,0.1$, and $1 \mathrm{nA}$ was injected at 10 -s intervals to test neuronal excitability. In some recordings, a second perfusion was performed to examine whether the effects of ap-GnRH could be washed out.

\section{ANALYSIS OF INTRACELLULAR ELECTROPHYSIOLOGICAL RECORDINGS}

A neuron was considered responsive to ap-GnRH if it exhibited one of the following three effects. First, an increase or a decrease of over $30 \%$ in maximum firing rate (MFR) was considered as excitatory or inhibitory, respectively. To assess MFR, the whole recording period $(10 \mathrm{~min})$ with either Veh or ap-GnRH treatment was divided into five blocks ( $2 \mathrm{~min} / \mathrm{block})$, and the spike number from a 10-s recording segment with the maximum firing frequency in each block counted. The sum of these 5 numbers represents the MFR of each neuron. Second, under ap-GnRH treatment, if a depolarizing current evoked a $>15 \%$ increase (excitatory) or decrease (inhibitory) in the total number of AP fired within the first $400 \mathrm{~ms}$ compared to Veh, the neuron was considered responsive to ap-GnRH. Lastly, after 1 min of treatment, if ap-GnRH resulted in a change of membrane potential over the pre-treatment baseline that was $200 \%$ greater than seen with Veh treatment, the neuron was considered responsive.

\section{STATISTICAL ANALYSIS}

Difference between treatment groups in the duration of $A D$, the number of compound $\mathrm{AP}$ fired during an $\mathrm{AD}$, and the number of stimulations needed to trigger an AD was assessed by the Student's $t$-test. Fisher's exact test was used to determine the difference in percent BCN clusters capable of firing an AD between Veh and ap-GnRH treatments. One way analysis of variance (ANOVA) followed by Tukey's post hoc test was used to compare the levels of ap-GnRH in nerves. Differences were considered significant when $P<0.05$.

\section{RESULTS}

\section{ap-GnRH NEURONS IN PEDAL GANGLIA PROJECTED TO NERVES INNERVATING THE FOOT AND PARAPODIA}

Figure 1 shows the anatomy of the A. californica CNS and the projection of relevant nerves ( $\mathrm{P} 1, \mathrm{P} 8$, and $\mathrm{P} 9)$ to the foot and parapodia. To determine if ap-GnRH neurons projected to the primary nerves responsible for the motor innervation of the foot and parapodia, pedal ganglia were labeled simultaneously via P1, $\mathrm{P} 8$, and $\mathrm{P} 9$ with a retrograde tracer, nickel chloride, followed by apGnRH ICC (Figure 2). In Figure 2A, four double-labeled neurons were observed. The nickel chloride labeling could be more clearly visualized in the same four neurons from the adjacent section (Figure 2B), which had not been processed for ap-GnRH ICC. Some ap-GnRH-immunoreactive (ir) neurons not labeled with nickel chloride were also observed (Figure 2A, asterisks), suggesting that not all ap-GnRH neurons projected to these nerves. The positions of four double-labeled neurons were in the superior (s) and medial (m) quadrant of pedal ganglia (Figure 2), consistent with the positions of ap-GnRH-ir neurons previously reported for these ganglia (Tsai et al., 2010). This experiment was repeated in three different animals. A total of 6,8 , and 10 double-labeled neurons were found in different individuals, respectively.

\section{ap-GnRH PEPTIDE WAS PRESENT IN EFFERENT MOTOR NERVE EXTRACTS}

Extracts of nerves P1, P8, or P9, which were known to be motor, contained high levels of ap-GnRH, whereas the extract of PVC, which had no known motor function, had a very low level of apGnRH (Figure 3). ap-GnRH level in PVC was significantly lower 


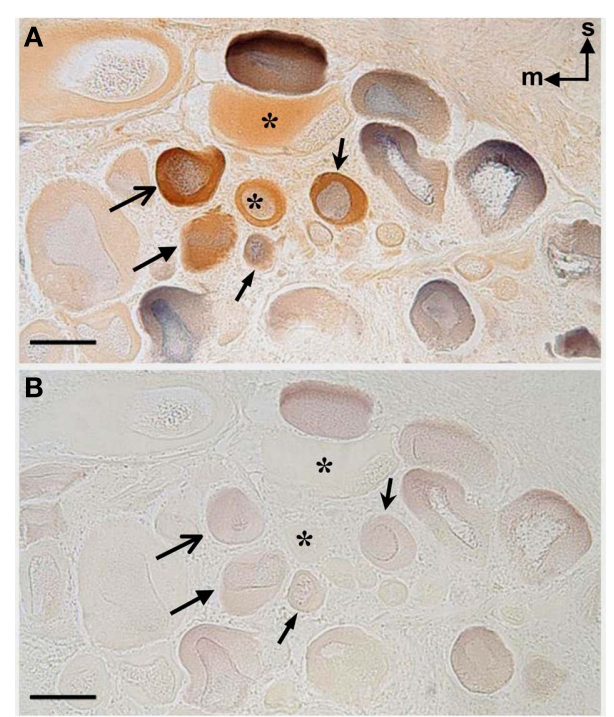

FIGURE 2 | Double-labeling of ap-GnRH neurons in the pedal ganglia of $\boldsymbol{A}$. californica by ICC and nickel backfill. $(\mathbf{A}, \mathbf{B})$ are adjacent sections. After nickel backfill, sections were processed for nickel color detection with (A) or without (B) ap-GnRH ICC. Superior (s) and medial (m) orientations are denoted in (A). Blue color, nickel backfill; brown color, ap-GnRH immunostaining. The four double-labeled neurons in $(\mathbf{A}, \mathbf{B})$ are labeled by arrows, and the same neurons are indicated by arrows of the same shape. In (A,B), * represents ap-GnRH neurons not labeled by nickel. Scale bars $=100 \mu \mathrm{m}$.

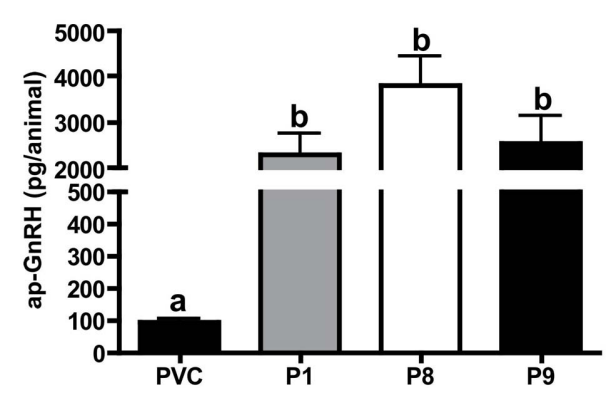

FIGURE 3 | Quantification of ap-GnRH peptide in nerve extracts by an ap-GnRH RIA. Each bar represents mean \pm SEM. $N=5$ for PVC, P8, and P9; $N=4$ for P1.

than that in $\mathrm{P} 1(P<0.05), \mathrm{P} 8(P<0.001)$, and P9 $(P<0.05)$, but there were no significant differences among extracts from different pedal nerves (Figure 3).

\section{ap-GnRH DECREASED THE LIKELIHOOD OF AD IN BCN}

Using extracellular recording of $\mathrm{BCN}$, we examined if ap-GnRH modulated the characteristics of $\mathrm{AD}$ and the ability of $\mathrm{BCN}$ to undergo an AD. The application of $73 \mathrm{nM}$ ap-GnRH to BCN clusters did not significantly alter the number of compound AP fired during an $\mathrm{AD}$ (Figure 4A), the duration of $\mathrm{AD}$ (Figure 4B) and the number of stimulations needed to trigger an $\mathrm{AD}$ (Figure $4 \mathrm{C}$ ) in sexually mature A. californica. However, ap-GnRH significantly decreased the number of responsive BCN clusters capable of initiating an $\mathrm{AD}$ under our stimulus protocol $(P=0.0188$, Figure 4D).

\section{ap-GnRH ALTERED ELECTROPHYSIOLOGICAL PROPERTIES OF CENTRAL NEURONS}

A total of 65 neurons have been recorded from buccal, cerebral, pedal, pleural, and abdominal ganglia. $29.2 \%$ of the recorded neurons were responsive to ap-GnRH treatment. The responses were diverse and included excitatory, inhibitory, and alteration of resting membrane potential. A summary of the effects was shown in Table 1.

Representative recording traces of different effects of ap-GnRH are shown in Figures 5-8. ap-GnRH $(730 \mathrm{nM})$ inhibited the spontaneous bursting of a neuron located on the inferior quadrant of the right abdominal ganglion (ventral surface; Figure 5). The regular bursting pattern exhibited by this neuron under Veh treatment (Figure 5, top) was suppressed with ap-GnRH (Figure 5, middle) but returned after a washout period (Figure 5, bottom). Not all neurons responded to ap-GnRH. For example, when treated with ap-GnRH, a neuron located on the superior quadrant of the right cerebral ganglion (ventral surface) remained quiescent with no apparent changes in firing pattern after current injections (Figure 6). Next, a neuron located on the superior quadrant of the left pedal ganglion (dorsal surface) was originally quiescent but responded to ap-GnRH with tonic firing and an increased firing rate after depolarization (Figure 7). Lastly, a neuron located on the lateral quadrant of the left pedal ganglion (ventral surface) was undergoing spontaneous tonic firing before treatment (Figure 8, top). The addition of ap-GnRH inhibited AP firing to reveal only the excitatory postsynaptic potential (EPSP; Figure 8, top and middle). The AP firing remained inhibited even after depolarization to a plateau potential by current injection of $1 \mathrm{nA}$.

\section{DISCUSSION}

Although the structure of multiple non-chordate GnRHs has recently been elucidated, many are still considered peptides in search of a function. At present, the primary physiological roles of these peptides have not yet emerged clearly from the limited functional studies (reviewed in Tsai and Zhang, 2008). The goal of the current study is therefore to fill some of these knowledge gaps by exploring the central functions of ap-GnRH. Our results show that ap-GnRH exerts diverse effects on all central ganglia and is directly positioned for the modulation of foot and parapodial movement. Unexpectedly, ap-GnRH reduces the likelihood of $\mathrm{BCN}$ clusters to undergo $\mathrm{AD}$, suggesting an inhibitory effect on female reproduction.

We previously showed that ap-GnRH injection enhanced parapodial opening and substrate attachment in A. californica, all of which could be accomplished by efferent input from the pedal ganglia to the foot and parapodia. In A. californica, the pedal nerve P1 innervates the anterior part of foot, and P8 innervates the middle part of foot. P9 innervates both the posterior parts of the foot and parapodia (Jahan-Parwar and Fredman, 1978). The uptake of nickel chloride by some ap-GnRH neurons from these nerves supported the direct projection of ap-GnRH neurons to these efferent nerves. Interestingly, in A. californica, the majority of motor neurons in the pedal ganglia were located medially and rostrally 

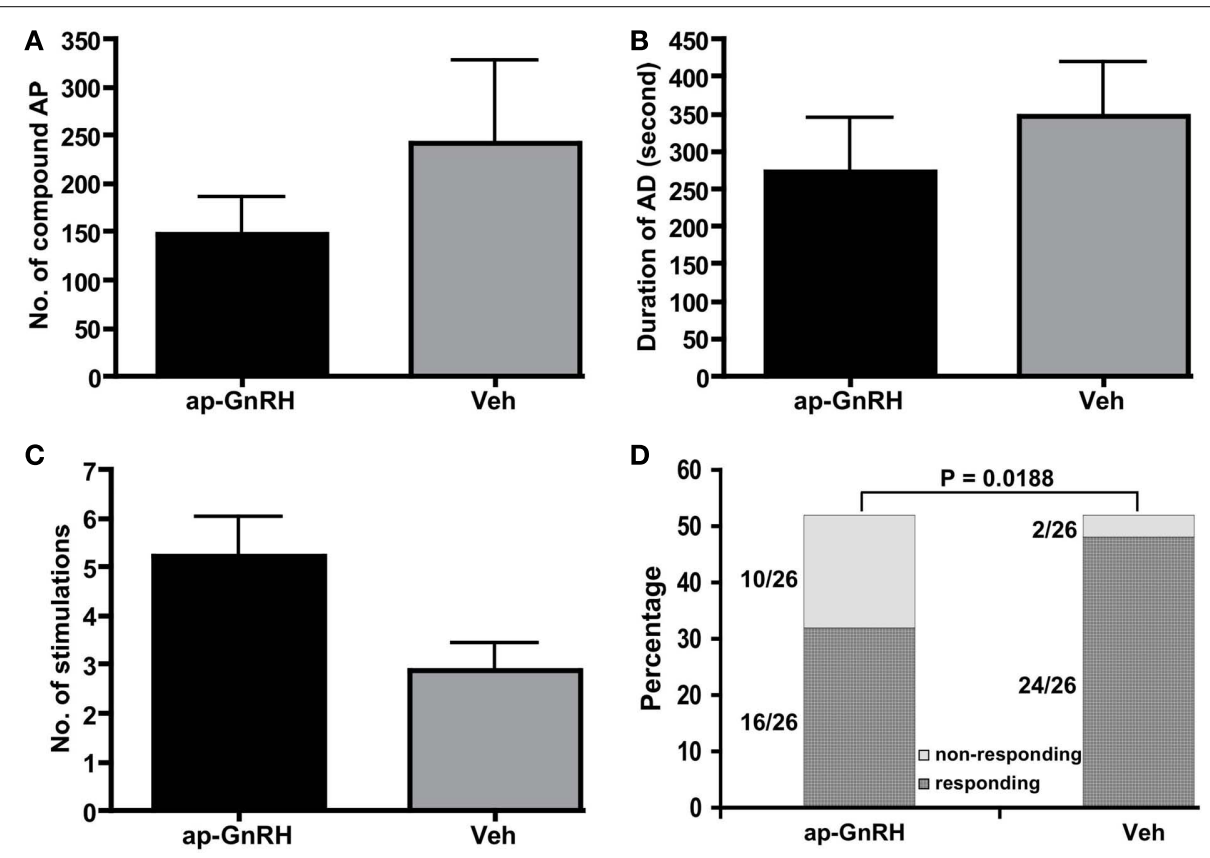

FIGURE 4 | Treatment of BCN clusters with $73 \mathrm{nM}$ ap-GnRH did not alter (A) the number of compound $A P$ fired during an $A D$, (B) the duration of $A D$ or (C) the number of stimulations needed to trigger an AD. (D) $73 \mathrm{nM}$ ap- $\mathrm{GnRH}$ decreased the number of bag cell clusters capable of undergoing an $A D$ $(P=0.0188) . N=26$ for all groups.

Table 1 | Percentage of effects in the central ganglia in response to the addition of $730 \mathrm{nM}$ ap-GnRH $(N=65)$.

\begin{tabular}{|c|c|c|c|c|c|c|}
\hline \multirow[t]{3}{*}{ Ganglia } & \multirow{3}{*}{$\begin{array}{l}\text { Number of } \\
\text { recordings }\end{array}$} & \multicolumn{5}{|c|}{ Effects } \\
\hline & & \multirow{2}{*}{$\begin{array}{l}\text { Excitatory (spikes only, } \\
\text { spontaneous, and evoked) (\%) }\end{array}$} & \multirow{2}{*}{$\begin{array}{l}\text { Inhibitory (spikes only, } \\
\text { spontaneous, and evoked) (\%) }\end{array}$} & \multicolumn{2}{|c|}{ Membrane potential change } & \multirow{2}{*}{$\begin{array}{l}\text { Non-responding } \\
(\%)\end{array}$} \\
\hline & & & & Depolarizing (\%) & Hyperpolarizing (\%) & \\
\hline Cerebral & 6 & 0 & 0 & 0 & 16.7 & 83.3 \\
\hline Abdominal & 15 & 6.7 & 20 & 0 & 20 & 53.3 \\
\hline Buccal & 8 & 0 & 12.5 & 0 & 0 & 87.5 \\
\hline Total & 65 & & & & & \\
\hline
\end{tabular}

(Hening et al., 1979), a location consistent with double-labeled neurons found in the present study. Further, motor neurons in A. brasiliana that innervate parapodial muscles to control swimming were also located in the same position of the pedal ganglia (McPherson and Blankenship, 1991). The neuroanatomical overlap between identified motor neurons and ap-GnRH neurons, coupled with the observation that ap-GnRH neurons project to efferent motor nerves (P1, P8, and P9), provided the first evidence that some ap-GnRH neurons may also function as motor neurons.

In addition to directly modulating the effector muscles, apGnRH may also affect motor functions via direct or trans-synaptic modulation of motor neurons. Indeed, we have observed a wide range of electrophysiological effects elicited by ap-GnRH within the CNS. A cognate receptor for ap-GnRH has not yet been identified, but the conserved nature of $\mathrm{GnRH}$ receptor (GnRHR) family across animal phyla suggests the receptor for ap-GnRH is likely a G-protein coupled receptor (GPCR) that couples to either Gq/11 or $G_{s}$ and utilizes inositol trisphosphate and diacylglycerol or cAMP as the primary second messengers (Millar et al., 2004). The cascades triggered by these pathways could be rather diverse and could explain the highly divergent effects seen in our intracellular recordings. In support of this notion, a wide range of electrophysiological effects, including excitatory, inhibitory, and alteration of input resistance, had also been observed when central neurons of the pond snails were treated with mGnRH (Goldberg et al., 1993). 


\section{Veh}

ap-GnRH

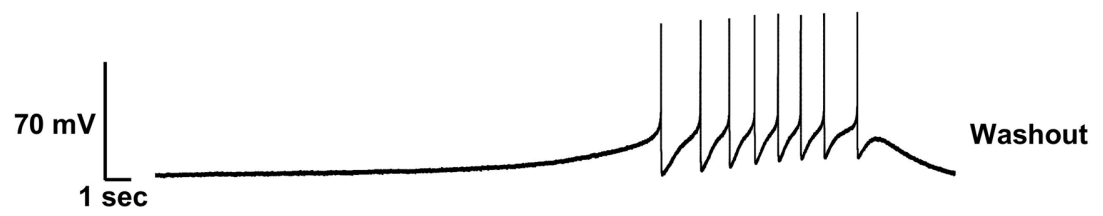

FIGURE 5 | Effect of ap-GnRH on a spontaneously bursting neuron located on the inferior quadrant of the right abdominal ganglion (ventral

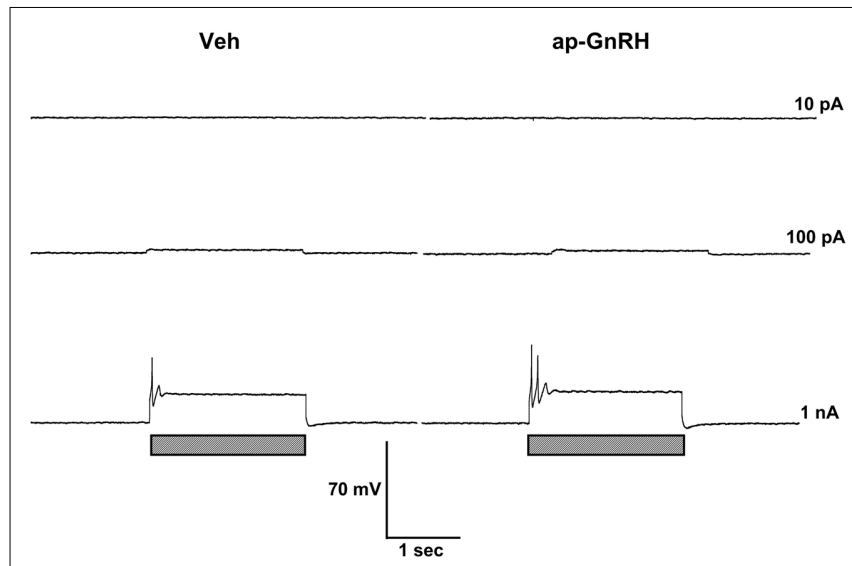

FIGURE 6 | ap-GnRH did not alter the firing pattern and the evoked AP of a neuron located on the superior quadrant of the right cerebral ganglion (ventral surface). Left panels represent recording traces of the neuron treated with Veh. Right panels represent the same neuron treated with $730 \mathrm{nM}$ ap-GnRH. Current injections (indicated by hatched bars on bottom) were given at $10 \mathrm{pA}$ (top), $100 \mathrm{pA}$ (middle), and $1 \mathrm{nA}$ (bottom).

The total levels of ap-GnRH in extracts of P1, P8, or P9 exceeded those previously reported for the whole pedal ganglia (3500 pg/animal; Tsai et al., 2010). This raised the question of whether pedal ganglia could supply enough ap-GnRH to the nerves, and whether these nerves synthesized ap-GnRH locally. A similar situation was observed for the pedal peptide (Pep) in $A$. californica, a peptide present at higher levels in the pedal nerves than the pedal ganglia proper (Hall and Lloyd, 1990; Pearson and Llody, 1990). However, Pep was conventionally synthesized in the pedal ganglia proper and subsequently transported to and accumulated in the nerves. Likewise, ap-GnRH could be accumulated in the nerves in a similar fashion. An interesting dichotomy was that Pep decreased foot tonus to cause foot to lose its grip of the surface). The addition of $730 \mathrm{nM}$ ap-GnRH inhibited the spontaneous bursting of the neuron, but the activity was recovered after a washout period.

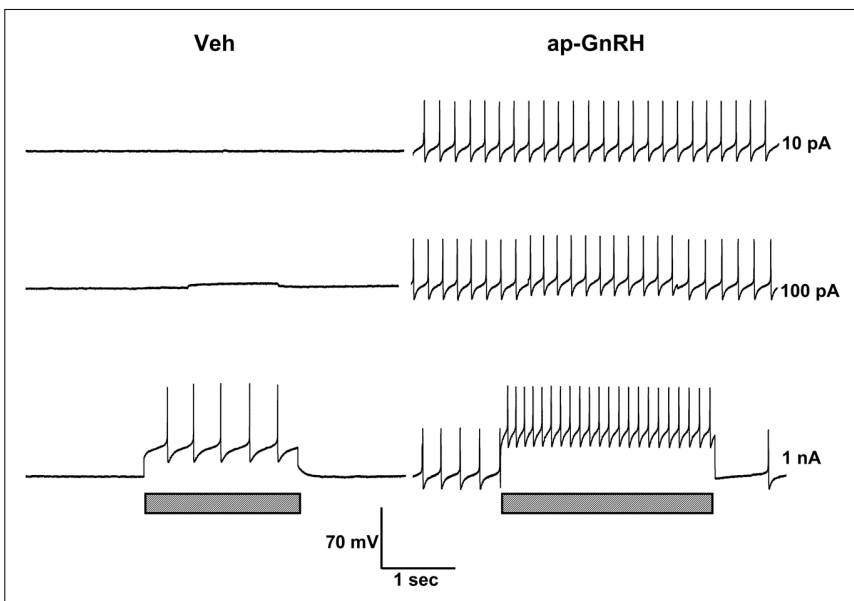

FIGURE 7 | ap-GnRH was excitatory to a neuron located on the superior quadrant of the left pedal ganglion (dorsal surface). The addition of $730 \mathrm{nM}$ ap-GnRH triggered the spontaneous firing (top, middle, and bottom before current injection) and increased the firing frequency of evoked AP when depolarized to a plateau potential (bottom). Left panels represent recording traces of the neuron treated with Veh. Right panels represent the same neuron treated with $730 \mathrm{nM}$ ap- $\mathrm{GnRH}$. Current injections (indicated by hatched bars on bottom) were given at $10 \mathrm{pA}$ (top) $100 \mathrm{pA}$ (middle), and $1 \mathrm{nA}$ (bottom).

substrate (Hall and Lloyd, 1990), whereas ap-GnRH increased foot tonus to improve substrate attachment (Tsai et al., 2010). It is tantalizing to hypothesize that both ap-GnRH and Pep are present in the same ganglia and nerves to coordinate different actions by pedal motor neurons on the effector muscles.

One way that ap-GnRH could exert the observed electrophysiological effects on the recorded neurons was by the direct modulation of ion channels and current types. In bullfrog sympathetic neurons, a well characterized model for GnRH peptidergic neurotransmission, $\mathrm{GnRH} 2$ promoted a late, slow EPSP by inhibiting 


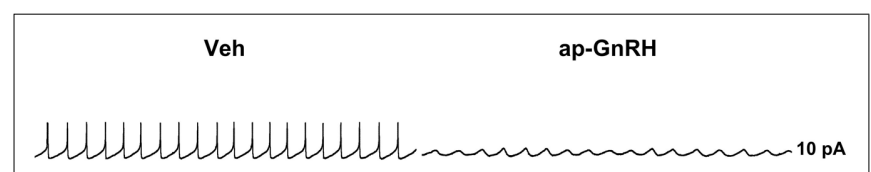

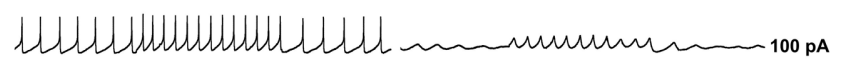

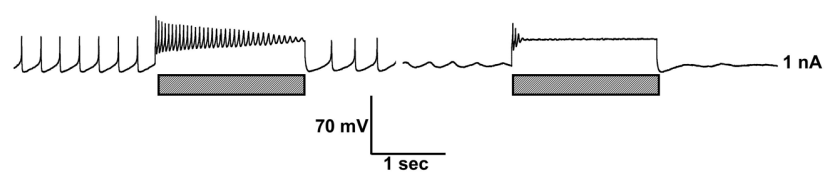

FIGURE 8 | The inhibitory effect of ap-GnRH on a neuron located on the lateral quadrant of the left pedal ganglion (ventral surface). The addition of $730 \mathrm{nM}$ ap-GnRH inhibited the spontaneous firing (top, middle, and bottom before current injection) and decreased evoked AP when depolarized to a plateau potential (bottom). Left panels represent recording traces of the neuron treated with Veh. Right panels represent the same neuron treated with $730 \mathrm{nM}$ ap-GnRH. Current injections (indicated by hatched bars on bottom) were given at $10 \mathrm{pA}$ (top), $100 \mathrm{pA}$ (middle), and $1 \mathrm{nA}$ (bottom).

a voltage-dependent $\mathrm{K}^{+}$current (Jones, 1987a,b). This enhanced the response to other synaptic inputs, decreased spike frequency adaption and promoted sustained repetitive firing (Jones, 1987b). GnRH2 also partially reduced the $\mathrm{N}$-type $\mathrm{Ca}^{2+}$ current and slowed the activity of the residual current (Elmslie et al., 1990). In addition to direct modulation, ap-GnRH could target the afferents of the recorded neurons to elicit trans-synaptic changes. As the neurocircuits governing movement are complex, it is at present difficult to identify these afferent targets, but the presence of ample sensory-motor connections and interneurons within the central ganglia certainly provides neural substrates for such regulation (Matsumoto et al., 1988; Bley and Tsien, 1990; Kawaski et al., 2007). Overall, ap-GnRH, through both direct and trans-synaptic mechanisms, can generate the diverse responses observed in our intracellular recordings.

In response to a brief stimulation from the cerebral ganglia or PVC, BCN can be depolarized to undergo an AD lasting for up to 30 min (Kupfermann and Kandel, 1970; Wayne and Wong, 1994). AD is a key driver for the release of ELH (Wayne and Wong, 1994), which in turn triggers ovulation and egg-laying behavior. Several peptides have been shown to modulate AD onset by altering ion channels and second messengers (reviewed in Conn and Kaczmarek, 1989). For example, peptide FMRFamide suppressed the initiation of the $\mathrm{AD}$ in response to electrical stimulation by

\section{REFERENCES}

Bley, K. R., and Tsien, R. W. (1990). Inhibition of $\mathrm{Ca}_{2}^{+}$and $\mathrm{K}^{+}$channels in sympathetic neurons by neuropeptides and other ganglionic transmitters. Neuron 4, 379-391.

Conn, P. J., and Kaczmarek, L. K. (1989). The bag cell neurons of Aplysia. Mol. Neurobiol. 3, 237-273.
Di Cosmo, A., and Di Cristo, C. the optic gland of Octopus vulgaris: FMRF-amide and GnRH immunoreactivity. J. Comp. Neurol. 398, 1-12. Di Cristo, C., De Lisa, E., and Di Cosmo, A. (2009). GnRH in the brain and ovary of Sepia officinalis. Peptides 30, 531-537. (1998). Neuropeptidergic control of

decreasing voltage-activated $\mathrm{Ca}^{2+}$ current and by activating an outward current carried by $\mathrm{K}^{+}$and $\mathrm{Cl}^{-}$(Fisher et al., 1993). Also, bag cell peptides (BCPs) were capable of depolarizing BCN by inducing a voltage-dependent inward $\mathrm{Na}^{+}$current (Loechner and Kaczmarek, 1994). Further, the second messenger cAMP could facilitate the initiation of an AD (Kaczmarek et al., 1978) in part by decreasing the delayed $\mathrm{K}^{+}$current and the A current (Kaczmarek and Strumwasser, 1981, 1984). We surmised that apGnRH could enhance or interfere with one of these mechanisms to inhibit the initiation of the AD. That said, since our BCN studies were performed in vitro, the overall reproductive impact of ap-GnRH was less clear. Our data suggested that $30.7 \%$ of BCN preparations became less likely to undergo an $\mathrm{AD}$ when treated with ap-GnRH. If we extrapolated this number to the whole animal, the negative reproductive consequence could be rather significant.

Although the primary role of $\mathrm{GnRH}$ in vertebrates is reproductive stimulation, there are ample reports on the non-reproductive roles of GnRH in both chordates and protostomes (Skinner et al., 2009). For example, GnRH modulates odorant response in the olfactory epithelium of the axolotl (Park and Eisthen, 2003) and regulates the development of the eye and brain in the zebrafish (Wu et al., 2006). In the O. vulgaris, GnRH promotes gonadal steroid synthesis and oviducal contraction (Iwakoshi-Ukena et al., 2004; Kanda et al., 2006), but also appears to be involved in movement and postural regulation unrelated to reproduction (Minakata et al., 2009). The diverse effects elicited by GnRH suggest the evolutionary trajectory of $\mathrm{GnRH}$ as a multifunctional neuropeptide.

In sum, the present study provides compelling evidence for a central role of ap-GnRH with links to motor modulation. Further, the negative effect of ap-GnRH on AD onset continues to support our previous views that ap-GnRH is not stimulatory to either BCN activity (Zhang et al., 2000) or egg-laying (Tsai et al., 2010). As such, we believe that the function of ap-GnRH, at least in A. californica, is not directed primarily toward reproductive stimulation. The direct projection of some ap-GnRH neurons to efferent motor nerves, the exceptional accumulation of ap-GnRH peptide in these nerves, and the ability of ap-GnRH to alter electrophysiological properties of diverse central neurons all point to what appear to be unconventional alternatives for the primary functions of this peptide.

\section{ACKNOWLEDGMENTS}

We appreciate Dr. Janet Casagrand's technical assistance on electrophysiological recordings, and Dr. Sue Moenter's helpful comments on data interpretation. This work was supported by NSF grant IOS 0743818 to Pei-San Tsai.

Di Cristo, C., and Di Cosmo, A. (2007). Neuropeptidergic control of Octopus oviducal gland. Peptides 28, 163-168.

Di Cristo, C., Paolucci, M., Iglesias, J., Sanchez, J., and Di Cosmo, A. (2002). Presence of two neuropeptides in the fusiform ganglion and reproductive ducts of Octopus vulgaris: FMRFamide and gonadotropin-releasing hormone (GnRH). J. Exp. Zool. 292, 267-276.

Elmslie, K. S., Zhou, W., and Jones, S. W. (1990). LHRH and GTP- $\gamma$-S modify calcium current activation in bullfrog sympathetic neurons. Neuron 5 , 75-80. 
Fisher, T., Lin, C. H., and Kaczmarek, L. K. (1993). The peptide FMRFa terminates a discharge in Aplysia bag cell neurons by modulating calcium, potassium, and chloride conductances. J. Neurophysiol. 69, 2164-2173.

Fredman, S. M. (1987). Intracellular staining of neurons with nickellysine. J. Neurosci. Methods 20, 181-194.

Goldberg, J. I., Garofalo, R., Price, C. J., and Chang, J. P. (1993). Presence and biological activity of a GnRHlike factor in the nervous system of Helisoma trivolvis. J. Comp. Neurol. 336, 571-582.

Hall, J. D., and Lloyd, P. E. (1990). Involvement of pedal peptide in locomotion in Aplysia: modulation of foot muscle contraction. J. Neurobiol. 21, 858-868.

Hening, W. A., Walters, E. T., Carew, T. J., and Kandel, E. R. (1979). Motorneuronal control of locomotion in Aplysia. Brain Res. 179, 231-253.

Iwakoshi, E., Takuwa-Kuroda, K., Fujisawa, Y., Hisada, M., Ukena, K., Tsutsui, K., and Minakata, H. (2002). Isolation and characterization of a GnRH-like peptide from Octopus vulgaris. Biochem. Biophys. Res. Commun. 291, 1187-1193.

Iwakoshi-Ukena, E., Ukena, K., TakuwaKuroda, K., Kanda, A., Tsutsui, K., and Minakata, H. (2004). Expression and distribution of octopus gonadotropin-releasing hormone in the central nervous system and peripheral organs of the octopus (Octopus vulgaris) by in situ hybridization and immunohistochemistry. J. Comp. Neurol. 477, 310-323.

Jahan-Parwar, B., and Fredman, S. M. (1978). Control of pedal and parapodial movements in Aplysia. I. Proprioceptive and tactile reflexes. $J$. Neurophysiol. 41, 600-608.

Jones, S. W. (1987a). Chicken II luteinizing hormone-releasing hormone inhibits the M-current of bullfrog sympathetic neurons. Neurosci. Lett. 80, 180-184.

Jones, S. W. (1987b). Luteinizing hormone-releasing hormone as a neurotransmitter in bullfrog sympathetic ganglia. Ann. N. Y. Acad. Sci. $519,310-322$.

Kaczmarek, L. K., Jennings, K., and Strumwasser, F. (1978). Neurotransmitter modulation, phosphodiesterase inhibitor effects, and cyclic AMP correlates of afterdischarge in peptidergic neuritis. Proc. Natl. Acad. Sci. U.S.A. 75, 5200-5204.

Kaczmarek, L. K., and Strumwasser, F. (1981). The expression of long lasting afterdischarge by isolated Aplysia bag cell neurons. J. Neurosci. 1, 626-634.

Kaczmarek, L. K., and Strumwasser, F. (1984). A voltage-clamp analysis of currents underlying cyclic AMP-induced membrane modulation in isolated peptidergic neurons of Aplysia. J. Neurophysiol. 52, 340-349.

Kah, O., Lethimonier, C., Somoza, G., Guilgur, L. G., Vaillant, C., and Lareyre, J. J. (2007). GnRH and GnRH receptors in metazoa: a historical, comparative, and evolutive perspective. Gen. Comp. Endocrinol. $153,346-364$

Kanda, A., Takahashi, T., Satake, H., and Minakata, H. (2006). Molecular and functional characterization of a novel gonadotropin-releasinghormone receptor isolated from the common octopus (Octopus vulgaris). Biochem. J. 395, 125-135.

Kandel, E. (1979). Behavioral Biology of Aplysia. San Francisco: W. H. Freeman and Company.

Kawaski, S., Kimura, S., Watanabe, S., Fujita, R., Matsumoto, M., and Sasaki, K. (2007). Augmenting effect of serotonin on the voltagedependent $\mathrm{Ca}^{+}{ }^{+}$current and subsequently activated $\mathrm{K}^{+}$current in Aplysia neurons. Tohoku J. Exp. Med. 211,31-41.

Kupfermann, I., and Kandel, E. R. (1970). Electrophysiological properties and functional interconnections of two symmetrical neurosecretory clusters (bag cells) in abdominal ganglion of Aplysia. J. Neurophysiol. $33,865-876$.

Loechner, K. J., and Kaczmarek, L. K. (1994). Autoactive peptides act at three distinct receptors to depolarize the bag cell neurons of Aplysia. J. Neurophysiol. 71, 195-203.

Matsumoto, M., Sasaki, K., Sato, M., Shozushima, M., and Takashima, K. (1988). Dopamine-induced depolarizing responses associated with negative slope conductance in LBcluster neurons of Aplysia. J. Physiol. 407, 199-213.

McPherson, D. R., and Blankenship, J. E. (1991). Neural control of swimming in Aplysia brasiliana. I. Innervation of parapodial muscle by pedal ganglion motoneurons. J. Neurophysiol. $66,1338-1351$.
Millar, R. P., Lu, Z. L., Pawson, A. J., Flanagan, C. A., Morgan, K., and Maudsley, S. R. (2004). Gonadotropin-releasing hormone receptors. Endocr. Rev. 25, 235-275.

Minakata, H., Shigeno, S., Kano, N. Haraguchi, S., Osugi, T., and Tsutsui, K. (2009). Octopus gonadotropinreleasing hormone: a multifunctional peptide in the endocrine and nervous systems of the cephalopod. J. Neuroendocrinol. 21, 322-326.

Nagle, G. T., Painter, S. D., Kelner, K. L., and Blankenship, J. E. (1985) Atrial gland cells synthesize a family of peptides that can induce egg laying in Aplysia. J. Comp. Physiol. B Biochem. Syst. Environ. Physiol. 156, 43-55.

Onitsuka, C., Yamaguchi, A., Kanamaru, H., Oikawa, S., Takeda, T., and Matsuyama, M. (2009). Molecular cloning and expression analysis of a GnRH-like dodecapeptide in the swordtip squid, Loligo edulis. Zool. Sci. 26, 203-208.

Park, D., and Eisthen, H. L. (2003). Gonadotropin releasing hormone $(\mathrm{GnRH})$ modulates odorant responses in the peripheral olfactory system of axolotls. J. Neurophysiol. 90, 731-738.

Pearson, W. L., and Llody, P. E. (1990). Distribution and characterization of pedal peptide immunoreactivity in Aplysia. J. Neurobiol. 21, 883-892.

Roch, G. J., Busby, E. R., and Sherwood, N. M. (2011). Evolution of GnRH: diving deeper. Gen. Comp. Endocrinol. 171, 1-16.

Skinner, D. C., Albertson, A. J., Navratil, A., Smith, A., Mignot, M., Talbott, H., and Scanlan-Blake, N. (2009) Effects of gonadotropin-releasing hormone outside the hypothalamicpituitary-reproductive axis. J. Neuroendocrinol. 21, 282-292.

Somoza, G. M., Miranda, L. A. Strobl-Mazzulla, P., and Guilgur, L. G. (2002). Gonadotropin-releasing hormone (GnRH): from fish to mammalian brains. Cell. Mol. Neurobiol. 22, 589-609.

Tsai, P. S., Maldonado, T. A., and Lunden, J. B. (2003). Localization of gonadotropin-releasing hormone in the central nervous system and a peripheral chemosensory organ of Aplysia californica. Gen. Comp. Endocrinol. 130, 20-28.

Tsai, P. S., Sun, B., Rochester, J. R., and Wayne, N. L. (2010). Gonadotropinreleasing hormone-like molecule is not an acute reproductive activator in the gastropod, Aplysia californica. Gen. Comp. Endocrinol. 166, 280-288.

Tsai, P. S., and Zhang, L. (2008). The emergence and loss of gonadotropin-releasing hormone in protostomes: orthology, phylogeny, structure, and function. Biol. Reprod. 79, 798-805.

Wayne, N. L., and Wong, H. (1994). Persistence of hormone secretion from neuroendocrine cells of Aplysia after termination of electrical after discharge. Endocrinology 134, 1046-1054.

Wu, S., Page, L., and Sherwood, N. M. (2006). A role for GnRH in early brain regionalization and eye development in zebra fish. Mol. Cell. Endocrinol. 257-258, 47-64.

Zhang, L., Tello, J. A., Zhang, W., and Tsai, P. S. (2008). Molecular cloning, expression pattern, and immunocytochemical localization of a gonadotropin-releasing hormone-like molecule in the gastropod mollusk, Aplysia californica. Gen. Comp. Endocrinol. 156, 201-209.

Zhang, L., Wayne, N. L., Sherwood, N. M., Postigo, H. R., and Tsai, P. S. (2000). Biological and immunological characterization of multiple GnRH in an opisthobranch mollusk, Aplysia californica. Gen. Comp. Endocrinol. 118, 77-89.

Conflict of Interest Statement: The authors declare that the research was conducted in the absence of any commercial or financial relationships that could be construed as a potential conflict of interest.

Received: 20 June 2011; paper pending published: 13 July 2011; accepted: 03 September 2011; published online: 20 September 2011.

Citation: Sun B and Tsai P-S (2011) A gonadotropin-releasing hormone-like molecule modulates the activity of diverse central neurons in a gastropod mollusk, Aplysia californica. Front. Endocrin. 2:36. doi: 10.3389/fendo.2011.00036

This article was submitted to Frontiers in Experimental Endocrinology, a specialty of Frontiers in Endocrinology. Copyright (c) 2011 Sun and Tsai. This is an open-access article subject to a nonexclusive license between the authors and Frontiers Media SA, which permits use, distribution and reproduction in other forums, provided the original authors and source are credited and other Frontiers conditions are complied with. 